\title{
Cloning and Differential Expression Analysis of a new $r b c S$ Gene from Lemna gibba
}

\author{
Youru Wang \\ ${ }^{1}$ Hubei Key Laboratory of Edible Wild Plant Conservation \& Utilization, Huangshi, Hubei, 435002, China \\ ${ }^{2}$ Life Science College, Hubei Normal University, Huangshi, Hubei, 435002, China
}

Corresponding author

\begin{abstract}
A novel Ribulose-1,5-bisphosphate carboxylase/oxygenase (Rubisco) small subunit gene (named ssu4d) was cloned from Lemna gibba by a novel chromosome walking technology, genomic DNA (gDNA) region of ssu4d with the size of $1,346 \mathrm{bp}$, was isolated. The full-length of ssu4d cDNA (named ssu4dc), contained a $522 \mathrm{bp}$ open reading frame encoding a protein of 174 amino acids, was also isolated. Sequence analysis of ssu $4 \mathrm{dc}$ and ssu $4 \mathrm{~d}$ showed that ssu4d contained an intron which is located from $+355 \mathrm{nt}$ to $+1125 \mathrm{nt}$ downstream transcriptional iniative site. ssu4dc contained 54 bp of $5^{\prime}$ untranslated region (UTR), and an open reading frame of 174 amino acids consisting of a chloroplast transit peptide with 57 amino acids and a mature protein of 117 amino acids. The deduced amino acid sequence of ssu4dc shared 95-96\% identity with L. gibba RbcS protein. Real time-PCR analysis showed differential expression of individual rbcS genes in light-grown Lemna gibba. And the levels of SSU4dc mRNA was regulated by the action of phytochrome, there was variability in the amount of expression of SSU4dc RNA comprared to the SSU1 and SSU5B from Lemna gibba.
\end{abstract}

KEYWORD: cloning; new rbcS gene; lemna gibba; transcriptional analysis

\section{INTRODUCTION}

Ribulose-1,5-bisphosphate carboxylase/oxygenase (Rubisco) which is the most abundant protein in nature catalyzes both the carboxylation of ribulose bisphosphate during $\mathrm{CO}_{2}$ fixation and the oxygenation of the same substrate during photorespiration (Carmo-Silva and Salvucci, 2011). Rubisco is currently the target enzyme for improving the efficiency of photosynthesis in the hope increasing the yield and growth of crops (Spreitzer, 2003). Meanwhile, the small subunits of Rubisco were produced by rbcS gene family in the nuclear genome, which are synthesized as $20-\mathrm{kDa}$ precursors in the cytoplasm and then processed to $15-\mathrm{kDa}$ during transport into the chloroplast (Thanh et al., 2011). In plants, the $55-\mathrm{kDa}$ large subunits are encoded by a single rbcL gene in the chloroplast genome(Spreitzer and Salvucci, 2002).The nucleotide and amino acid sequences of the RbcS vary among different organisms, while the primary structure of the large subunits is largely conserved (Whitney and Andrews, 2001; Mueller-Cajar and Whitney, 2008).

The duckweed Lemna gibba is an aquatic monocot in which the $r b c S$ is encoded by a 12-to 14member gene family (Buzby et al., 1990). The transcription of this gene family and the mRNA level of at least six of the genes have been shown to be regulated by phytochrome, genomic clones for six members of the gene family and a cDNA clone for a seventh have been isolated and characterized (Silverthorne et al., 1990; Stiekema et al., 1983). Each genomic sequence isolated contains a single intron at the position of the second intron of dicots (Silverthorne et al., 1990). The introns vary in length but have a high degree of homology with one another, whereas the 3' untranslated regions are sufficiently divergent over their full length to allow their use as gene-specific probes. Using genespecific probes, the expression of individual $\mathrm{rbcS}$ sequences in total steady state RNA was shown to be under the control of phytochrome (Silverthorne et al., 1990). However, there is considerable variation in the levels of expression of individual $\mathrm{rbcS}$ genes in two different organs: the roots and fronds. This variation could be explained by transcriptional differences, in some post-transcriptional event(s) (Silverthorne and Tobin, 1990). The most extreme differences between transcription rates versus steady state mRNA levels were measured for SSU1 and SSU5B (Acevedo-Hernandez et al., 2005).

In an effort to increase our knowledge of lemna gibba photosynthesis and elucidate relationship 
among $r b c S$ genes from lemna gibba, a new $r b c S$ gene (ssu4d) was isolated from Lemna gibba. The isolation of cloned ssu4d gene of the Lemna rbcS family has allowed us to examine the expression of these closely related genes and to determine whether there are differences in the effect of phytochrome action on individual family member.

\section{MATERIALS AND METHODS}

\subsection{Plant materials and growth conditions}

Axenic cultures of duckweed (Lemna gibba) were maintained in $150 \mathrm{ml}$ flasks containing $50 \mathrm{ml} \mathrm{SH}$ basal medium supplemented with $1 \%$ sucrose and cultured in a growth chamber at $28^{\circ} \mathrm{C}$ either under constant fluorescent lighting (90-150 $\mu \mathrm{mol}$ photons $\mathrm{m}^{-2} \mathrm{~s}^{-1}$ ) with a photoperiod of $16 \mathrm{~h}$ of light and $8 \mathrm{~h}$ of dark. Dark-grown duckweeds were harvested using

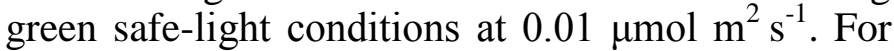
light-pulses, Duckweeds were treated with FR and RL pulses pulse of $2 \mathrm{~min}$ and $10 \mathrm{~min}$ reapectively. FR and RL pulses were at 120, $140 \mu \mathrm{mol} \mathrm{m} \mathrm{m}^{2} \mathrm{~s}^{-1}$ respectively. Duckweeds were harvested for total RNA extraction $24 \mathrm{~h}$ after the onset of the last irradiation under a dim green safelight.
2.2 Cloning and Sequence analysis of L. gibba rbcS gene

Genomic DNA was isolated from fronds of L. gibba using the modified CTAB extraction method (Luo et al., 2006). The PCR products were purified using QIAquick Gel Extraction Kit (Qiagen, Germany). Based on the known $L$. gibba $r b c S$ gene sequence, the generacy primers (PF1 and PR1) were designed (table 1) to obtain about 400 bp DNA fragment by PCR, This DNA fragment was cloned into the pMD18-T vector (TakaRa) for sequencing, as the 400 bp DNA fragment contained the 5' sequence of the new $r b c S$ gene, and the 3' region of the new rbcS gene was obtained by self-formed PCR (Wang et al., 2007) and the primers (sp1, sp2, sp3) were listed (table1. By bioinformatics analysis, series of 5 , forwars primers (PF2a, PF2b and PF2c) were designed to confirmed the transcriptional iniation site of $s s u 4 d c$ gene (table 1). The primers (table 1) was designed according to the cloned DNA sequence and used for cloning of cDNA of ssu4d (Table 1). The PCR fragments were cloned into the pMD18-T vector (TaKaRa, Dalian, China), the positive clones were sequenced. The DNA sequences were analyzed using DNAMAN5 software. Similarity searches were done online at the nucleotide level with BLASTn and at amino acid level with BLASTp.

Table 1. Primers used for cloning of $r b c S$ gene from L.gibba

\begin{tabular}{|l|l|l|}
\hline Primers & Primers sequence ( 5' to 3') & Purpose \\
\hline PF1 & MGATAAGRTGTAATCCW & cloning of $r b c S$ gene \\
\hline PR1 & TGGAAGCCATCATCGACGAAGCCAT & from $L$. gibba \\
\hline PF2a & AGAGACCCCAGAGCTTCCGAG & series of forward primers used for cloning of cDNA of \\
PF2b & GAGACCCCAGAGCTTCCGAG & ssu \\
PF2c & CAGAGACCCCAGAGCTTCCGAG & reverse primer for cloning of $s s u 4 d c$ cDNA \\
\hline PR2 & TTAGGTGGGCTTGTAGGCGATGAAG & 3'-walking \\
\hline SP1 & GCTTCGTCGATGATGGCTTCCACCG & 3'-walking \\
\hline SP2 & CGGCGACGAGGAAGGCTAACGATCTGT & 3'-walking \\
\hline SP3 & GGGGCTGAAGAAGTTCGNNNNNNNNNNCTACCT & \\
\hline
\end{tabular}

\subsection{Real-time PCR analysis of rbcS genes}

Total Lemna gibba RNA was isolated from L.gibba using a Trizol reagent (Invitrogen) following the manufacturer's instructions. Single-strand cDNA was synthesized from $2 \mu \mathrm{g}$ total RNA from each sample by reverse transcription (RT)-PCR using an RT Kit (TaKaRa, Dalian, China) according to the manufacture's instruction. Each real-time PCR reaction (15 $\mu$ l total volume) contained $2 \mu \mathrm{l}$ of template (cDNA), $7.5 \mu$ of $2 \times$ Sensi Mix (Quantace, London, UK), $0.3 \mu$ of $50 \times$ SYBR Green I Solution (Quantace), $0.33 \mu \mathrm{M}$ of each of the forward and reverse primers and ultrapure $\mathrm{H}_{2} \mathrm{O}$. The product of ssu4dc cDNA was amplified with two specific primers (ssu4d-TF: 5'- AGAGACCCCAGAGCTTC CGAGAAGA-3' as forward primer and ssu4d-TR: 5'-AGCCGTTGCGGAGGAGGTAGTCG-3' reverse primer), the product of ssu5B cDNA with 5B-F(5'-TGGCTTCGTCGATGATGGCTTCCAC3) and 5B-R (5'- GTTGTGGAAAGACCACGGGGAT ACG $\left.-3^{\prime}\right)$; the product of ssulcDNA with $1-\mathrm{F}$ (5'TTTCCACCGCCGCCGTGGCCCGCGT-3') and 1R (5'-GAATGACAGATACTTTTTGATTTAT -3'); using specific primers actin-F (5'-ATCCAATTCTC CTCACTGAAGCCCC-3') and actin-R (5'GGGGCTTCAGTGAGGAGAATTGGAT-3') as control. Reactions were performed in a Step-one plus real-time PCR system (applied biosystem). Initial denaturation was performed at $95^{\circ} \mathrm{C}$ for 10 minutes, followed by 45 cycles of: denaturation at $95^{\circ} \mathrm{C}$ for 30 seconds; annealing at $55^{\circ} \mathrm{C}$ for 30seconds; and extension at $72^{\circ} \mathrm{C}$ for 60 seconds. Each sample was run in triplicate in two separate reactions generating six readings per gene. 


\subsection{Isolation of the ssu4d gene from Lemna gibba}

There is a conserved region found in rbcS genes of Lemna gibba (GenBank accession No. X17231.1, X17230.1， X17232.1， X17235.1， X17234.1 X17233.1, and X00137.1). A pair of generacy primers were designed based on this region. A putative novel rbcS gene about $400 \mathrm{bp}$ fragment was obtained and sequenced, and eventually, a new rbcS gene with a length of $1346 \mathrm{bp}$ (designated as ssu4d) was cloned from L. gibba genomic DNA by SEFAPCR. Sequence blast analysis revealed that ssu4d shared 95-96 \% identity with the known L. gibba $\mathrm{rbcS}$ genes. According to the sequence of this putative novel $r b c S$ gene, specific primers were designed to amplify the $r b c S$ gene cDNA, transcriptional site was confirmed by designing a series of 5'forward primers based on the putative transcriptional site which was inferred by the analysis of known $r b c S$ genes from L gibba. A 579 bp $r b c S$ gene cDNA fragment was first generated by RT-PCR. Sequence analysis of this cDNA fragment showed ssu4dc shared 95-96 \% identity with Lemna gibba rbcS genes. The integrity of the genomic DNA (1346 bp in length) and cDNA (579 bp in length) sequences of ssu $4 \mathrm{~d}$ was confirmed by nest PCR.

\subsection{Sequence Analysis of L. gibba ssu4d gene}

Comparison of gDNA and cDNA sequences of ssu4d indicated that the position of the single intron in ssu4d gene was located from $+355 \mathrm{nt}$ to $+1125 \mathrm{nt}$ downstream the transcriptional iniation site. The intron, with the size of $770 \mathrm{nt}$, was quite different from the other intron of $\mathrm{L}$. gibba $\mathrm{rbcS}$ gene in length; the 5' splice site of the intron is TT and the 3' splice site is AC, which indicated that ssu4d contained different splicing mechnasim. cDNA fragment of 579 bp was analysed and searched against NCBI database to find the conserved domain and putative function. It was found that the Lemna gibba cDNA (ssu4dc) possessed a 522 bp open reading frame (ORF) from $55 \mathrm{bp}$ to $579 \mathrm{bp}$ of the sequence apart from a $54 \mathrm{bp} 5$ 'untranslated region (5'-UTR) which was underlined (figure. 1), and this cDNA encoded for a protein of which those domains were found similar to other L. gibba RbcS (figure. 1). The deduced amino acid sequences of ssu $4 \mathrm{~d}$ gene are shown (figure. 1) .The transit peptide is less conserved than the mature SSU polypeptide when the deduced amino acid sequence of ssu4dc from L.gibba was compared with those sequences from other organisms in GenBank database using BLASTX search. The result indicated that ssu4d conserved segments have broad homology to the cloned L. gibba $\mathrm{RbcS}$ with the similarity and identity.

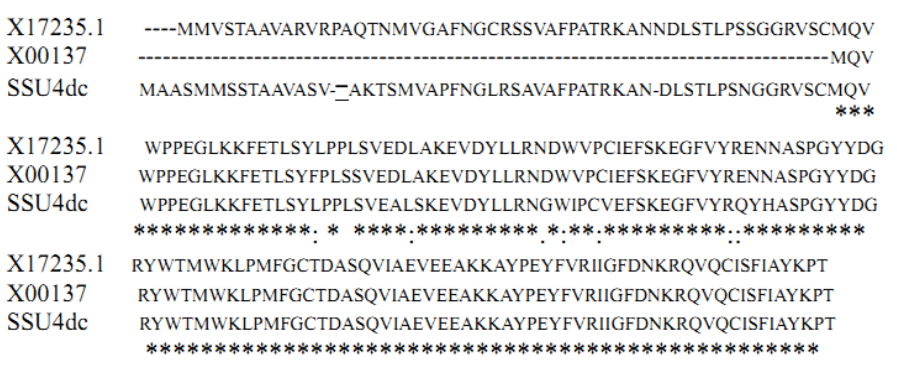

Fig.1 Comparison of Lemna gibba $r b c S$ sequences encoding the mature SSU protein. The deduced amino acid sequence of X17235.1, X00137 from L.gibba are shown.The deduced transient peptide of Ssu4dc was underlined with boldfaced letters. An asterisk(*) denotes the position of residues identical with X17235.1.

\subsection{Differential expression analysis of $r b c S$ gene}

The 5 '-UTR (untranslated region) and 3 '- UTR of rbcS genes from Lemna gibba represent distinct sequences according to the sequence comparison analysis. This made it possible to use such sequence to analyze the expression of specific genes. It was demonstrated that the ssul sequence is the most highly represented, followed by ssu5B in Lemna gibba (Silverthorne et al., 1990). In an attempt to examine the expression of ssu4dc, Real-time PCR analysis of the cDNA clone were performed using primers designed based on the 5'- UTR and 3 'UTR. Our results indicated that the SSU1 was the most highly represented, followed by ssu4dc and SSU5B, the expression level of ssu4dc gene was higher than that of SSU5B in light condition (Fig. 2).

Since the rbcS genes were expressed in white light-grown plants, we determined whether the different expression of $\mathrm{rbcS}$ gene under phytochrome regulation. The cDNA clone was used to template to evaluate the different expression of $\mathrm{rbcS}$ gene. The results of real-time PCR analysis demonstrated that the expression of each rbcS gene could be increased by red light pulses, although the magnitude of this response varies widely between the genes (Fig. 3). The responses to red light can be reversed by far-red light(Fig. 3). Although the expressed levels of the SSU40A, SSU40B and ssu4dc mRNAs are very different, they could also be demonstrated to be phytochrome-regulated.

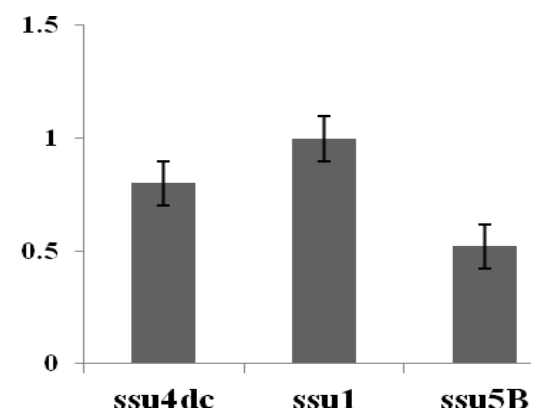

Fig. 2. Differential expression of individual $r b c S$ genes in lightgrown plants. The amount of expression of each gene is normalized to the expression of SSUI. The mean of triplicate determinations is shown. 


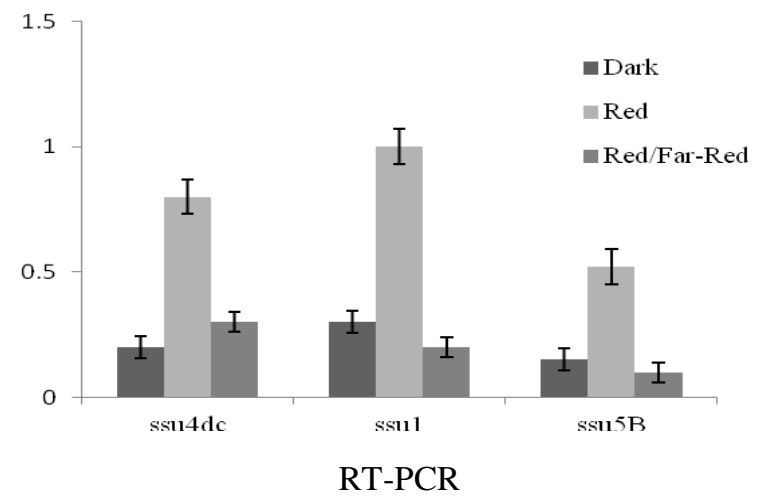

Fig. 3 phytochrome-regulated expression of individual $r b c S$ genes. The relative expression levels of SSU4dc, SSU1 and SSU5B are shown in arbitrary units. Dark, Red, and Red/Farred refer to the growth conditions described in Materials and methods. The mean of triplicate determinations is shown.

\section{DISCUSSION}

In this paper, a novel L. gibba rbcS gene (ssu4d), which was 1,346 bp long, was cloned from L. gibba, and the isolated 579-bp cDNA of ssu4d (ssu4dc) contained a 522-bp open reading frame encoding a protein of 174 amino acids, BLAST revealed that the cloned cDNA sequence of ssu4dc was highly similar to the rbcS genes from other species and the deduced ssu4dc protein also showed high identity to the RBCS proteins from other plant species via multialignments. Bioinformatics analysis showed that SSU4dc protein might belong to the rbcS supergene family. There was only one intron located between +355 and +1225 , which indicated that ssu4dc gene was quite different from the cloned rbcS genes from duckweed. ssu4dc contained 54 bp of 5' untranslated region (UTR), and an open reading frame of 174 amino acids consisting of a chloroplast transit peptide with 57 amino acids and a mature protein of 117 amino acids. The deduced amino acid sequence of ssu4dc shared 95-96\% identity with Lemna gibba RBCS.

Quantitative analysis indicated that SSU4dc mRNA accumulated to much lower levels than SSU1 mRNA in steady state nuclear mRNA isolated from fronds, but higher levels than SSU5B mRNA. The expression levels of the SSU1, SSU5B and ssu4dc mRNAs varied very different, we could not rule out the possibility that the differential expression of individual $\mathrm{rbcS}$ genes is the result of more than one regulatory event. The analysis of these regulatory steps may provide a clue as to the mechanisms of phytochrome regulation of $\mathrm{rbcS}$ gene expression.

\section{REFERENCES}

[1] Acevedo-Hernandez, G.J., Leon, P., Herrera-Estrella, L.R., 2005. Sugar and ABA responsiveness of a minimal
RBCS light-responsive unit is mediated by direct binding of ABI4. Plant J 43, 506-519.

[2] Bartholomew, D.M., Bartley, G.E., Scolnik, P.A., 1991. Abscisic Acid Control of rbcS and cab Transcription in Tomato Leaves. Plant Physiol 96, 291-296.

[3] Buzby, J.S., Yamada, T., Tobin, E.M., 1990. A lightregulated DNA-binding activity interacts with a conserved region of a Lemna gibba rbcS promoter. Plant Cell 2, 805-814.

[4] Carmo-Silva, A.E., Salvucci, M.E., 2011. The activity of Rubisco's molecular chaperone, Rubisco activase, in leaf extracts. Photosynthesis research 108, 143-155.

[5] Gray, J.C., Sullivan, J.A., Wang, J.H., Jerome, C.A., MacLean, D., 2003. Coordination of plastid and nuclear gene expression. Philos Trans R Soc Lond B Biol Sci 358, 135-145.

[6] Huijser, C., Kortstee, A., Pego, J., Weisbeek, P., Wisman, E., Smeekens, S., 2000. The Arabidopsis SUCROSE UNCOUPLED-6 gene is identical to ABSCISIC ACID INSENSITIVE-4: involvement of abscisic acid in sugar responses. Plant J 23, 577-585.

[7] Laby, R.J., Kincaid, M.S., Kim, D., Gibson, S.I., 2000. The Arabidopsis sugar-insensitive mutants sis4 and sis5 are defective in abscisic acid synthesis and response. Plant J 23, 587-596.

[8] Luo, K., Zheng, X., Chen, Y., Xiao, Y., Zhao, D., McAvoy, R., Pei, Y., Li, Y., 2006. The maize Knotted1 gene is an effective positive selectable marker gene for Agrobacterium-mediated tobacco transformation. Plant Cell Rep 25, 403-409.

[9] Mueller-Cajar, O., Whitney, S.M., 2008. Directing the evolution of Rubisco and Rubisco activase: first impressions of a new tool for photosynthesis research. Photosynthesis research 98, 667-675.

[10] Rolland, F., Baena-Gonzalez, E., Sheen, J., 2006. Sugar sensing and signaling in plants: conserved and novel mechanisms. Annu Rev Plant Biol 57, 675-709.

[11] Silverthorne, J., Wimpee, C.F., Yamada, T., Rolfe, S.A., Tobin, E.M., 1990. Differential expression of individual genes encoding the small subunit of ribulose-1,5bisphosphate carboxylase in Lemna gibba. Plant Mol Biol $15,49-58$.

[12] Spreitzer, R.J., 2003. Role of the small subunit in ribulose-1,5-bisphosphate carboxylase/oxygenase. Archives of biochemistry and biophysics 414, 141-149.

[13] Spreitzer, R.J., Salvucci, M.E., 2002. Rubisco: structure, regulatory interactions, and possibilities for a better enzyme. Annual review of plant biology 53, 449-475.

[14] Stiekema, W.J., Wimpee, C.F., Tobin, E.M., 1983. Nucleotide sequence encoding the precursor of the small subunit of ribulose 1,5-bisphosphate carboxylase from Lemna gibba L.G-3. Nucleic Acids Res 11, 8051-8061.

[15] Thanh, T., Chi, V.T., Abdullah, M.P., Omar, H., Noroozi, M., Napis, S., 2011. Cloning and characterization of ribulose-1,5-bisphosphate carboxylase/oxygenase small subunit (RbcS) cDNA from green microalga Ankistrodesmus convolutus. Mol Biol Rep 38, 52975305.

[16] Wang, S., He, J., Cui, Z., Li, S., 2007. Self-formed adaptor PCR: A simple and efficient method for chromosome walking. Appl Environ Microbiol.

[17] Weatherwax, S.C., Ong, M.S., Degenhardt, J., Bray, E.A., Tobin, E.M., 1996. The interaction of light and abscisic acid in the regulation of plant gene expression. Plant physiology 111, 363-370.

[18] Whitney, S.M., Andrews, T.J.,2001. The gene for the ribulose 1,5-bisphosphate carboxylas (Rubisco) small subunit relocated to the plastid genome of tobacco directs the synthesis of small subunits that assemble into Rubisco. The Plant cell 13, 193-205. 